\title{
Direct synthesis of parallel doped N-MoP/N-CNT as highly active hydrogen evolution reaction catalyst
}

\author{
Juntao Zhang ${ }^{1}$, Rui Sui ${ }^{1}$, Yanrong Xue ${ }^{1}$, Xingdong Wang ${ }^{1}$, Jiajing Pei ${ }^{1}$, Xin Liang ${ }^{2,3^{*}}$ and \\ Zhongbin Zhuang ${ }^{1,3 *}$
}

\begin{abstract}
Doped phosphide is promising in earthabundant element based catalysts for hydrogen evolution reaction (HER). Here we employ ammonium hypophosphite $\left(\mathrm{NH}_{4} \mathrm{H}_{2} \mathrm{PO}_{2}\right)$ to synthesize a novel parallel doped catalyst, nitrogen doped molybdenum phosphide nanoparticles (NPs) supported on nitrogen doped carbon nanotubes $(\mathrm{N}-\mathrm{MoP} /$ $\mathrm{N}$-CNTs). The $\mathrm{NH}_{4} \mathrm{H}_{2} \mathrm{PO}_{2}$ as a bifunctional agent severs as both phosphidation agent and nitrogen source, which makes the synthetic route simple and efficient. The as-obtained parallel doped $\mathrm{N}-\mathrm{MoP} / \mathrm{N}-\mathrm{CNT}$ show an overpotential of $103 \pm 5 \mathrm{mV}$ at $10 \mathrm{~mA} \mathrm{~cm}^{-2}$, which is $140 \mathrm{mV}$ lower than that of MoP NPs. The enhanced HER performance is attributed to the electronic effect by doped MoP and CNTs supports. This work provides a facile route to synthesize doped phosphides for the potential applications in hydrogen energy.
\end{abstract}

Keywords: bifunctional precursor, ammonium hypophosphite, nitrogen-doped MoP, nitrogen-doped carbon nanotubes, hydrogen evolution reaction

\section{INTRODUCTION}

Hydrogen, with high energy density and zero polluted gas emission, has been considered as an ideal candidate for renewable, greenhouse-gas-free and sustainable energy [1,2-4]. Electrochemical water splitting coupled with renewable electricity is an ideal technique for sustainably realizing hydrogen production [5-7]. Pt-based electrocatalysts show the best electrocatalytic activity for hydrogen evolution reaction (HER) [8,9]. However, the high cost and scarcity of Pt limit their wide applications. Therefore, efficient noble-metal-free cathode materials for HER gained much attention in the past few years [1016]. The pristine non-precious metals cannot survive in acidic environment, so the research mainly focuses on their compounds. Transition metal phosphides are found promising for HER [12,14,17-21]. In particular, molybdenum phosphide (MoP), a recently discovered nonprecious metal phosphide HER catalyst, has attracted intensive attention because of its good electrocatalytic activity and stability [22-26]. And their potential HER performance could be realized by fine tuning the structure of the catalysts.

Doping is considered as an effective way to improve the electrochemical activities [27-31]. Doping the MoP catalyst can tune the electronic structure of the active sites. Nitrogen doped [32], sulfur doped [33], or nitrogen and sulfur co-doped [34] MoP were reported to have better HER activities than the intrinsic MoP. Chan et al. [35] theoretically studied the doping effect of MoP, and they found that the hydrogen binding energy of MoP can be tuned by the dopant, leading to higher HER activity. Doping the support was also found to be beneficial for the electrocatalyst by the synergistic effect, because the commonly used carbon supports have strong interaction with the catalyst nanoparticles (NPs) [36-38]. Nitrogendoped carbon supported MoP NPs enhanced HER performance $[39,40]$. Simultaneously doping both catalyst NPs and support might further improve the catalytic performance. However, the commonly used doping method is to introduce the doping agent in the synthesis, which makes the synthetic procedure complicated.

In this paper, we developed a novel bifunctional agent, ammonium hypophosphite $\left(\mathrm{NH}_{4} \mathrm{H}_{2} \mathrm{PO}_{2}\right)$, which can be used as both the phosphidation agent and also the nitrogen doping source in one reaction. Sodium hypophosphite [19,41] and ammonium phosphate [39] have been used previously. However, they can only be used as single functional agent. By annealing carbon

\footnotetext{
${ }^{1}$ State Key Laboratory of Organic-Inorganic Composites, Beijing University of Chemical Technology, Beijing 100029, China

${ }^{2}$ State Key Laboratory of Chemical Resource Engineering, Beijing University of Chemical Technology, Beijing 100029, China

${ }^{3}$ Beijing Key Laboratory of Energy Environmental Catalysis, Beijing University of Chemical Technology, Beijing 100029, China

* Corresponding authors (emails: liangxin@mail.buct.edu.cn (Liang X); zhuangzb@mail.buct.edu.cn (Zhuang Z))
} 
nanotubes $(\mathrm{CNT})$ supported molybdenum oxides $\left(\mathrm{MoO}_{x} /\right.$ CNTs) precursor with $\mathrm{NH}_{4} \mathrm{H}_{2} \mathrm{PO}_{2}$ in a sealed and evacuated tube, we successfully synthesized a novel parallel doped catalyst, N-doped MoP NPs supported on N-doped CNTs (N-MoP/N-CNTs). The as-obtained parallel doped N-MoP/N-CNTs catalyst has an overpotential of $103 \pm 5 \mathrm{mV}$ at $10 \mathrm{~mA} \mathrm{~cm}^{-2}$, and a low Tafel slope of $42 \mathrm{mV} \mathrm{dec}^{-1}$. The high HER activity is attributed to the tuned electronic structure by doping $\mathrm{N}$ in $\mathrm{MoP}$ NPs and the synergistic effect by coupling the NPs with N doped CNTs. This is a simple method to dope the catalyst and support with $\mathrm{N}$ via phosphidation, and the parallel doped N-MoP/N-CNTs electrocatalyst has a great potential to replace the noble catalyst for HER.

\section{EXPERIMENTAL SECTION}

\section{Materials}

$\mathrm{NH}_{4} \mathrm{H}_{2} \mathrm{PO}_{2}, \quad\left(\mathrm{NH}_{4}\right)_{6} \mathrm{Mo}_{7} \mathrm{O}_{24} \cdot 4 \mathrm{H}_{2} \mathrm{O}$, ethylene glycol, $\mathrm{NaNO}_{3}, \quad \mathrm{NaH}_{2} \mathrm{PO}_{2}, \mathrm{KMnO}_{4}$ were purchased from Sinopharm Chemical Reagent Beijing Ltd. $\mathrm{H}_{2} \mathrm{O}_{2}, \mathrm{HCl}$, $\mathrm{H}_{2} \mathrm{SO}_{4}$ (98\%), were purchased from Beijing Chemical Factory. CNTs were purchased from Cnano Technology Ltd. Pt/C (20 wt\%) and Nafion dispersion (5 wt\%) was purchased from Alfa Aesar. All the chemicals were used as received without further purification. Deionized water $(18.2 \mathrm{M} \Omega \mathrm{cm})$ was used throughout all experiments.

\section{Catalyst synthesis}

\section{Synthesis of $\mathrm{MoO}_{x} / \mathrm{CNTS}$}

The CNTs were firstly oxidized through modified Hummer method [36]. $20 \mathrm{mg}$ of oxidized CNTs were dissolved in $4 \mathrm{~mL}$ of deionized water and sonicated at least $30 \mathrm{~min}$ to form a homogeneous dispersion, and then $50 \mathrm{mg}$ of $\left(\mathrm{NH}_{4}\right)_{6} \mathrm{Mo}_{7} \mathrm{O}_{24} \cdot 4 \mathrm{H}_{2} \mathrm{O}$ was added and sonicated for $10 \mathrm{~min}$, followed by the addition of $16 \mathrm{~mL}$ of ethylene glycol and another $30 \mathrm{~min}$ of sonication. Then the dispersion was poured into a $40 \mathrm{~mL}$ Teflon-lined autoclave. The autoclave was sealed and placed in an oven for hydrothermal processing at $200^{\circ} \mathrm{C}$ for $24 \mathrm{~h}$. After natural cooling to room temperature, the black precipitate was collected by centrifugation and washed three times with ethanol and deionized water, respectively. Then the product was frozen and lyophilized overnight for further experiments and characterizations.

\section{Synthesis of N-MoP/N-CNTs}

$20 \mathrm{mg}$ of $\mathrm{MoO}_{x} / \mathrm{CNT}$ and $1 \mathrm{~g}$ of $\mathrm{NH}_{4} \mathrm{H}_{2} \mathrm{PO}_{2}$ were put into two ceramic boats respectively, and then transferred into a quartz tube. The quartz tube was bubbled by $\mathrm{N}_{2}$, evacuated and finally sealed. Then, it was heated to $800^{\circ} \mathrm{C}$ with a slow heating rate of $2.5^{\circ} \mathrm{C} \mathrm{min}^{-1}$, and kept at this temperature for $2 \mathrm{~h}$. The sample was collected after cooling to the ambient temperature.

\section{Synthesis of the control samples}

The control samples, including MoP/CNTs, MoP NPs and N-CNTs were synthesized according to the similar procedure to fabricate $\mathrm{N}-\mathrm{MoP} / \mathrm{N}-\mathrm{CNT}$. MoP/CNTs were synthesized by replacing $\mathrm{NH}_{4} \mathrm{H}_{2} \mathrm{PO}_{2}$ to $\mathrm{NaH}_{2} \mathrm{PO}_{2}$. For MoP NPs, $\mathrm{NaH}_{2} \mathrm{PO}_{2}$ was used without CNTs. For NCNTs, CNTs was heated with $\mathrm{NH}_{4} \mathrm{H}_{2} \mathrm{PO}_{2}$.

\section{Characterizations}

The as-synthesized samples were characterized by X-ray powder diffraction (XRD) using a Bruker D8 diffractometer with $\mathrm{Cu} \mathrm{Ka}$ radiation $(\lambda=0.15406 \mathrm{~nm})$. The morphology of the sample was investigated by a transmission electron microscope (TEM, FEI Quanta 250) operating at $200 \mathrm{kV}$. High resolution TEM (HRTEM) images and energy dispersive X-ray spectroscopy (EDX) elemental mapping profiles were obtained on Tecnai G2F20 S-TWIN electron microscope. The Xray photoelectron spectroscopy (XPS) was carried out on a Kratos Axis Ultra DLD spectrometer using an $\mathrm{Al} \mathrm{Ka}$ monochromated ( $h v=1486.6 \mathrm{eV}, 150 \mathrm{~W})$. Thermogravimetric analysis (TGA) was investigated on Mettler Toledo TGA1 connected to infrared (IR) analyzer (ThermoFisher 6700) to investigate the evolved gases.

The electrochemical performances were evaluated on CHI 660E electrochemical workstation using a standard three-electrode system. The HER tests were performed in $0.5 \mathrm{~mol} \mathrm{~L}^{-1} \mathrm{H}_{2} \mathrm{SO}_{4}$. Graphite rod was used as the counter electrode to avoid the $\mathrm{Pt}$ contamination and saturated calomel electrode was used as the reference electrode. The reference electrode was calibrated with respect to reversible hydrogen electrode (RHE) in this report and the zero point of RHE was calibrated using the equilibrium potential of $\mathrm{HOR} / \mathrm{HER}$ of $\mathrm{Pt}$ catalyst in $\mathrm{H}_{2}$ saturated electrolyte. All the measurements reported in this paper were after $i R$ correction. The catalyst deposited on the glassy carbon electrode was severed as the working electrode. The catalyst ink was made by dispersing $2 \mathrm{mg}$ catalyst powder in $500 \mu \mathrm{L}$ mixed solvent $\left(380 \mu \mathrm{L} \mathrm{H}_{2} \mathrm{O}\right.$, $100 \mu \mathrm{L}$ ethanol and $20 \mu \mathrm{L}$ Nafion dispersion (5 wt $\%)$ ). Then the mixture was sonicated for at least $1 \mathrm{~h}$ to obtain homogeneous ink. $20 \mu \mathrm{L}$ of the ink was transferred onto a polished glassy carbon electrode $(d=5 \mathrm{~mm})$, resulting in a loading of $0.41 \mathrm{mg} \mathrm{cm}^{-2}$. The HER performance was 

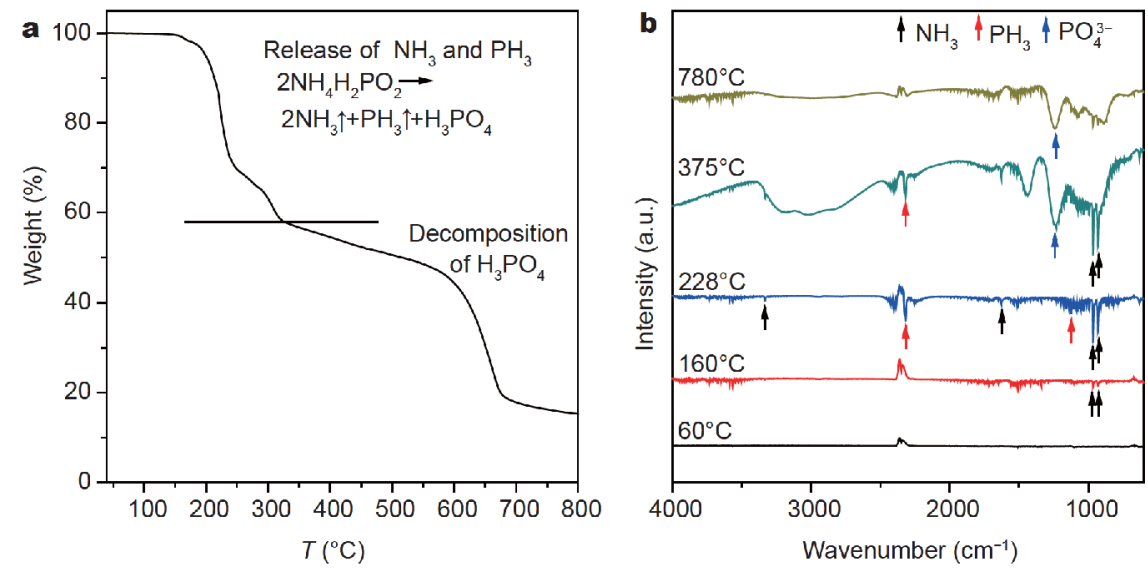

Figure 1 The decomposition of $\mathrm{NH}_{4} \mathrm{H}_{2} \mathrm{PO}_{2}$. (a) TGA curve of $\mathrm{NH}_{4} \mathrm{H}_{2} \mathrm{PO}_{2}$ tested under $\mathrm{N}_{2}$ atmosphere. (b) The corresponding IR spectra of the evolved gas during the TGA test.

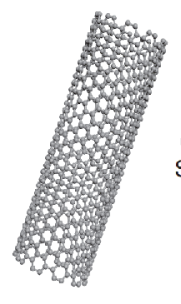

CNT

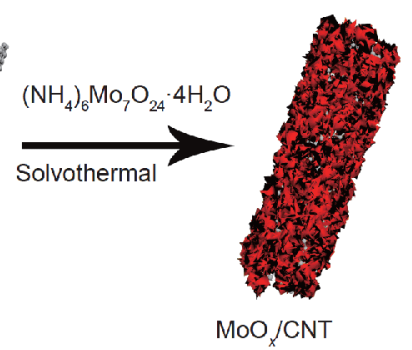

$\mathrm{MOO} / \mathrm{CNT}$

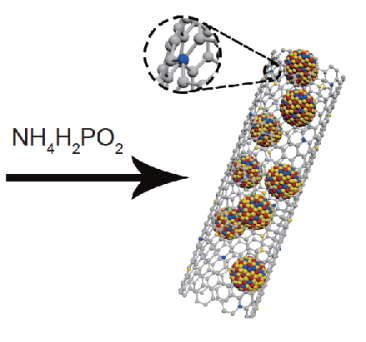

N-MOP/N-CNT

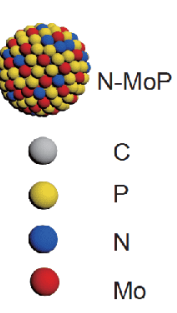

Mo

Figure 2 The schematic of the formation of N-MoP/N-CNT.

measured by linear sweep voltammetry (LSV) at a sweep rate of $5 \mathrm{mV} \mathrm{s}^{-1}$ in $\mathrm{H}_{2}$ saturated electrolyte. The electrochemical impedance spectroscopy (EIS) was carried out at the frequency from $0.1 \mathrm{~Hz}$ to $100 \mathrm{kHz}$.

\section{RESULTS AND DISCUSSION}

$\mathrm{NH}_{4} \mathrm{H}_{2} \mathrm{PO}_{2}$ is the key agent for the phosphidation and the nitrogen doping. $\mathrm{NH}_{4} \mathrm{H}_{2} \mathrm{PO}_{2}$ decomposes upon heating to disproportionally generate $\mathrm{PH}_{3}$ which works as the phosphidation agent. $\mathrm{NH}_{3}$ was also released, which served as the nitrogen doping agent. Fig. 1a shows the TGA curve of $\mathrm{NH}_{4} \mathrm{H}_{2} \mathrm{PO}_{2}$ under $\mathrm{N}_{2}$ flow, and the evolved gases were tested by Fourier transform infrared spectrometer (FTIR, Fig. 1b). $\mathrm{NH}_{4} \mathrm{H}_{2} \mathrm{PO}_{2}$ started to decompose at around $160^{\circ} \mathrm{C}$ and released ammonia confirmed by the typical $\mathrm{N}-\mathrm{H}$ vibration located at 3,330, 1,625, 970 and $933 \mathrm{~cm}^{-1}$ [42]. When the temperature increased to $225^{\circ} \mathrm{C}$, $\mathrm{PH}_{3}$ was detected, indicated by $\mathrm{P}-\mathrm{H}$ vibration located at 2,320 and $1,125 \mathrm{~cm}^{-1}$ [43]. $\mathrm{PH}_{3}$ came from the disproportionation of hypophosphite, with phosphoric acid generated at the same time. Phosphoric acid is unstable upon heating, and it condensed to form polyphosphoric acids, followed by the gradual evaporation, which was demonstrated by the gradual weight loss and the $\mathrm{P}-\mathrm{O}$ vibration located at $1,236 \mathrm{~cm}^{-1}$ in the evolved gas [43]. Thus, $\mathrm{NH}_{4} \mathrm{H}_{2} \mathrm{PO}_{2}$ decomposes as Equations (1-3):

$$
\begin{gathered}
\mathrm{NH}_{4} \mathrm{H}_{2} \mathrm{PO}_{2} \rightarrow \mathrm{NH}_{3}+\mathrm{H}_{3} \mathrm{PO}_{2}, \\
2 \mathrm{H}_{3} \mathrm{PO}_{2} \rightarrow \mathrm{PH}_{3}+\mathrm{H}_{3} \mathrm{PO}_{4}, \\
\mathrm{H}_{3} \mathrm{PO}_{4} \rightarrow \mathrm{HPO}_{3}+\mathrm{H}_{2} \mathrm{O} .
\end{gathered}
$$

$\mathrm{NH}_{3}$ and $\mathrm{PH}_{3}$ are both generated by heating $\mathrm{NH}_{4} \mathrm{H}_{2} \mathrm{PO}_{2}$, so it is a bifunctional agent that can be used as both phosphidation agent and nitrogen doping agent.

$\mathrm{N}-\mathrm{MoP} / \mathrm{N}-\mathrm{CNT}$ was synthesized by incorporation of $\mathrm{NH}_{4} \mathrm{H}_{2} \mathrm{PO}_{2}$ through a simple two step route. As shown in Fig. 2, $\mathrm{MoO}_{x}$ was first coated onto CNTs via solvothermal method by using ammonium molybdate $\left(\left(\mathrm{NH}_{4}\right)_{6} \mathrm{Mo}_{7} \mathrm{O}_{24} \cdot 4 \mathrm{H}_{2} \mathrm{O}\right)$ as the Mo source and water and ethylene glycol mixture as the solvent. Then the precursor was heated with $\mathrm{NH}_{4} \mathrm{H}_{2} \mathrm{PO}_{2}$ in a sealed tube which was 

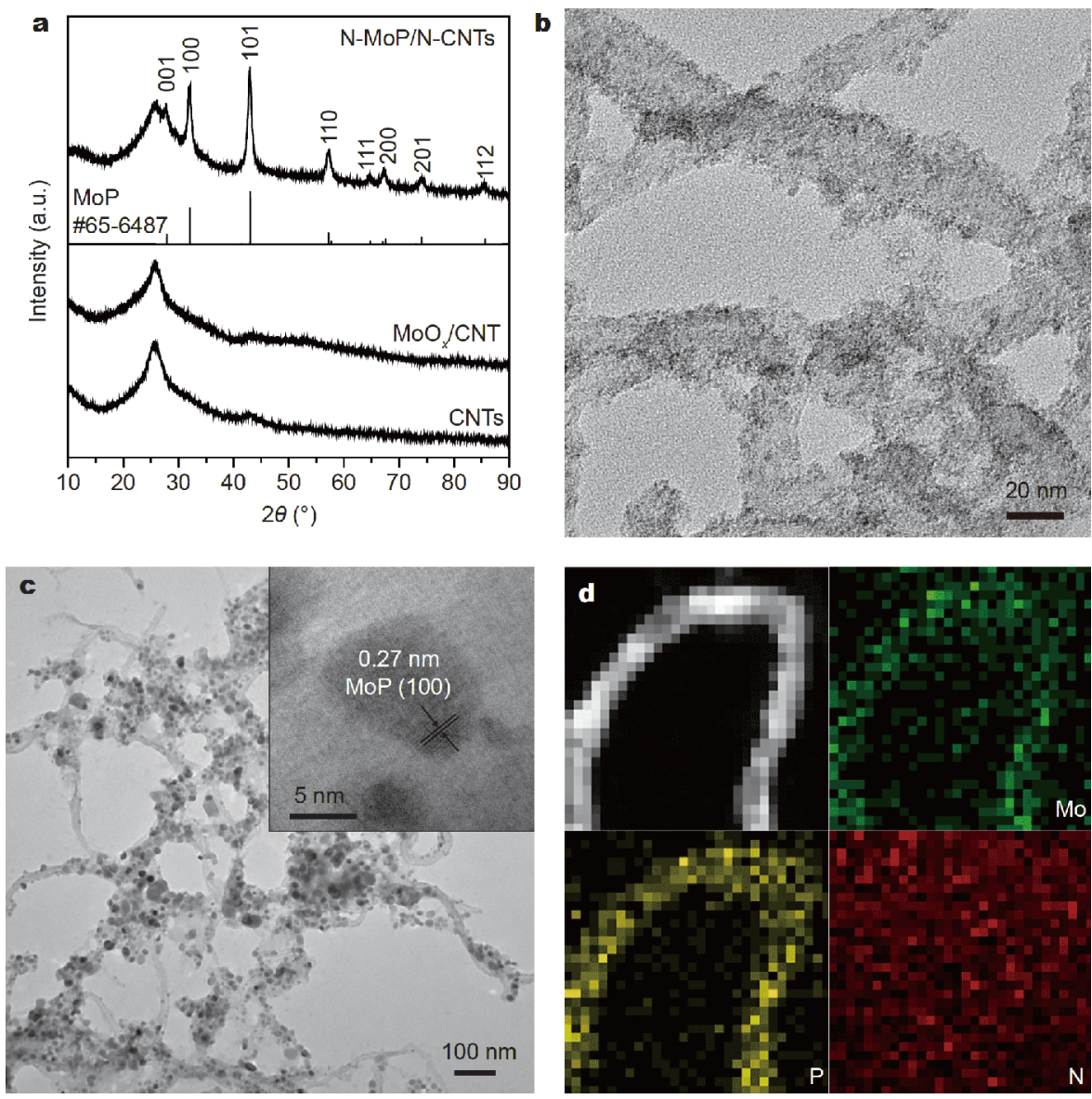

Figure 3 Characterization of the as-obtained N-MoP/N-CNTs. (a) XRD patterns of $\mathrm{MoO}_{x} / \mathrm{CNT}$ and N-MoP/N-CNTs. (b, c) TEM images of MoO ${ }_{x} /$ CNTs and N-MoP/N-CNTs; inset: HRTEM image of N-MoP/N-CNTs, (d) STEM images and EDX mapping of Mo, P and N of N-MoP/N-CNTs.

previously evacuated. By using this bifunctional agent, the coated $\mathrm{MoO}_{x}$ transformed into MoP NPs supported on CNTs, and MoP and CNTs were doped with $\mathrm{N}$ at the same time.

Fig. 3a shows the XRD patterns of the $\mathrm{MoO}_{x} / \mathrm{CNTs}$ precursor and $\mathrm{N}-\mathrm{MoP} / \mathrm{N}-\mathrm{CNT}$ catalyst. Only one major peak assigned to CNTs was shown for the $\mathrm{MoO}_{x} / \mathrm{CNTs}$ precursor, indicating the existence of the amorphous structure of the coated $\mathrm{MoO}_{x}$. After the phosphidation process, additional reflection peaks assigned to MoP (JCPDS card No. 65-6487) revealed the successful conversion of $\mathrm{MoO}_{x}$ to MoP. The TEM image (Fig. S1) indicates the obtained oxidized nanotubes are highly dispersed with a diameter of about $20 \mathrm{~nm}$. After reacting with ammonium molybdate, the obtained $\mathrm{MoO}_{x} / \mathrm{CNTs}$ precursor shows a rough tubular surface (Fig. 3b), which is considered as the coated amorphous $\mathrm{MoO}_{x}$. After the phosphidation process at high temperature, $\mathrm{MoO}_{x}$ layers transform into MoP NPs to form $\mathrm{N}-\mathrm{MoP} / \mathrm{N}-\mathrm{CNTs}$, as shown in Fig. 3c. The CNTs maintain the structure after the high temperature treatment, and the MoP NPs, with diameter of 8-15 nm, distributed on the CNTs uniformly. The HRTEM image (inset of Fig. 3c) further reveals the lattice fringe with spacing of $0.27 \mathrm{~nm}$, which corresponds to the (100) plane of MoP. The EDX mapping profiles for $\mathrm{N}-\mathrm{MoP} / \mathrm{N}-\mathrm{CNT}$ s show that the Mo, $\mathrm{N}$ and $\mathrm{P}$ are distributed in CNTs uniformly in Fig. 3d.

We also investigated the effect of the amount of Mo source used in the synthesis on the morphologies of $\mathrm{N}$ MoP/N-CNTs. As shown in Fig. S2, with the amount of the Mo source increasing, more MoP NPs formed on the CNTs. However, too much Mo source used in the synthesis resulted in large particle size of the MoP on CNTs. Thus the optimal amount of the Mo source was identified. MoP NPs and MoP/CNTs are also synthesized for comparison, and their XRD patterns and TEM images are shown in Figs S3 and S4.

XPS was adopted to explore the surface composition 

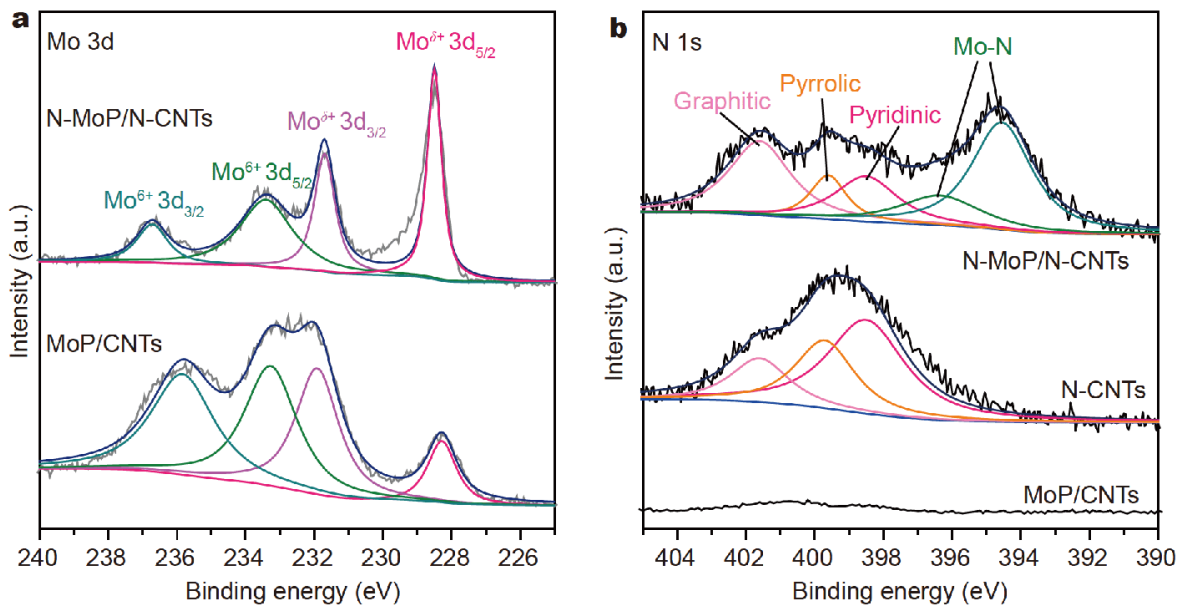

Figure 4 High resolution core level XPS spectra. (a) Mo 3d spectra of N-MoP/N-CNTs and MoP/CNTs. (b) N 1s spectra of N-MoP/N-CNTs, NCNTs and MoP/CNTs.

and valence states of the N-MoP/N-CNTs. The survey of $\mathrm{N}-\mathrm{MoP} / \mathrm{N}-\mathrm{CNTs}$ (Fig. S5a) confirms the existence of Mo, $\mathrm{P}$, and N. Fig. 4a shows the Mo $3 \mathrm{~d}$ core level spectra of NMoP/N-CNTs. The peaks at 228.5 and $231.8 \mathrm{eV}$ are assigned to the $\mathrm{Mo}\left(\delta^{+}\right) \quad 3 \mathrm{~d}_{5 / 2}$ and $3 \mathrm{~d}_{3 / 2}$ of $\mathrm{MoP}$, respectively [44]. Compared with the $\operatorname{Mo}\left(\delta^{+}\right) 3 d_{5 / 2}$ peak of MoP/CNT $(228.3 \mathrm{eV})$, there is a positive shift of $0.2 \mathrm{eV}$ for the N-MoP/N-CNTs, which is ascribed to the high electronegativity of doped N. And other two peaks at 236.7 and $233.5 \mathrm{eV}$ are assigned to $\mathrm{Mo}^{6+}$ due to the surface oxidation [25]. For the P $2 p$ core level spectra (Fig. S5b), two peaks at 129.3 and $130.7 \mathrm{eV}$ can be assigned to $\mathrm{P}-\mathrm{Mo}$ species in N-MoP/N-CNTs at $2 \mathrm{p}_{3 / 2}$ and $2 \mathrm{p}_{1 / 2}$, and the peak at $134.0 \mathrm{eV}$ can be assigned to the oxidized species $\left(\mathrm{PO}_{4}{ }^{3-}\right.$ or $\left.\mathrm{P}_{2} \mathrm{O}_{5}\right)$ owing to exposure to the air [45]. Fig. $4 \mathrm{~b}$ shows the $\mathrm{N} 1 \mathrm{~s}$ core level spectra of MoP/ CNTs, N-CNTs and N-MoP/N-CNTs. No N signal was observed for MoP/CNTs. For N-CNTs, a broad peak in the range of $395-405 \mathrm{eV}$ was observed, which can be deconvoluted into three peaks at 398.9, 399.7 and $401.6 \mathrm{eV}$, corresponding to pyridinic, pyrrolic and graphitic $\mathrm{N}$, respectively, confirming that the $\mathrm{N}$ atoms were doped into the CNTs. For N-MoP/N-CNTs, similar $\mathrm{N}$ species from $\mathrm{N}-\mathrm{CNT}$ was observed, and the doping level was calculated as 8.3 at\% according to the XPS results. In addition, there are two peaks at 397.5 and $394.6 \mathrm{eV}$ assigned to the $\mathrm{N}-\mathrm{Mo}$ species in $\mathrm{N}-\mathrm{MoP} / \mathrm{N}-$ CNTs $[34,46]$, which confirms the successful doping of nitrogen into MoP. For the $\mathrm{C}$ 1s core level spectra (Fig. S5c), there are two peaks at 284.8 and $286.0 \mathrm{eV}$ which are ascribed to $\mathrm{C}-\mathrm{C}(\mathrm{C}=\mathrm{C})$ and $\mathrm{C}-\mathrm{N}(\mathrm{C}=\mathrm{N})$ [47], respectively, also suggesting the successful incorporation of $\mathrm{N}$ into the CNTs.

The electrochemical activity of the as-synthesized N$\mathrm{MoP} / \mathrm{N}-\mathrm{CNTs}$ catalyst towards HER was investigated in $\mathrm{H}_{2}$-saturated $0.5 \mathrm{~mol} \mathrm{~L}^{-1} \mathrm{H}_{2} \mathrm{SO}_{4}$ using a standard threeelectrode system. MoP/CNTs, MoP NPs, N-CNTs and CNTs were also studied under the same conditions. All the potentials reported in this paper are versus RHE with the $i R$ compensation. The catalyst loadings on the glassy carbon electrode were controlled at $0.41 \mathrm{mg} \mathrm{cm}^{-2}$. Fig. $5 \mathrm{a}$ shows the polarization curves of as-prepared samples with a sweep rate of $5 \mathrm{mV} \mathrm{s}^{-1}$. The CNTs and N-CNTs show poor HER performance. The MoP NPs can be used as HER catalyst, but with low activity. When MoP is supported on CNTs, the HER performance is promoted. MoP/CNTs show an overpotential of $129 \mathrm{mV}$ at $10 \mathrm{~mA} \mathrm{~cm}^{-2}$, which is lower than that of MoP NPs $(243 \mathrm{mV})$. The promoted HER activity is attributed to uniform dispersion of the MoP NPs on CNTs and also the good electronic conductivity of CNTs. When parallel doped the MoP NPs and CNTs with N, the HER performance was further improved. $\mathrm{N}-\mathrm{MoP} / \mathrm{N}-\mathrm{CNTs}$ only needs an overpotential of $103 \pm 5 \mathrm{mV}$ (the error is the standard deviation obtained at five independent experiments) at $10 \mathrm{~mA} \mathrm{~cm}^{-2}$, which is 26 and $140 \mathrm{mV}$ smaller than MoP/CNTs and MoP NPs, respectively. The HER performance of all the catalysts are summarized in Table 1. It demonstrates that doping with $\mathrm{N}$, both in the CNTs substrate and the MoP NPs, is beneficial to HER. These parallel doped N-MoP/N-CNTs also show good HER performance compared with the reported MoPbased HER catalysts (Table S1). It was found that $800^{\circ} \mathrm{C}$ was the optimal temperature for the synthesis of the 

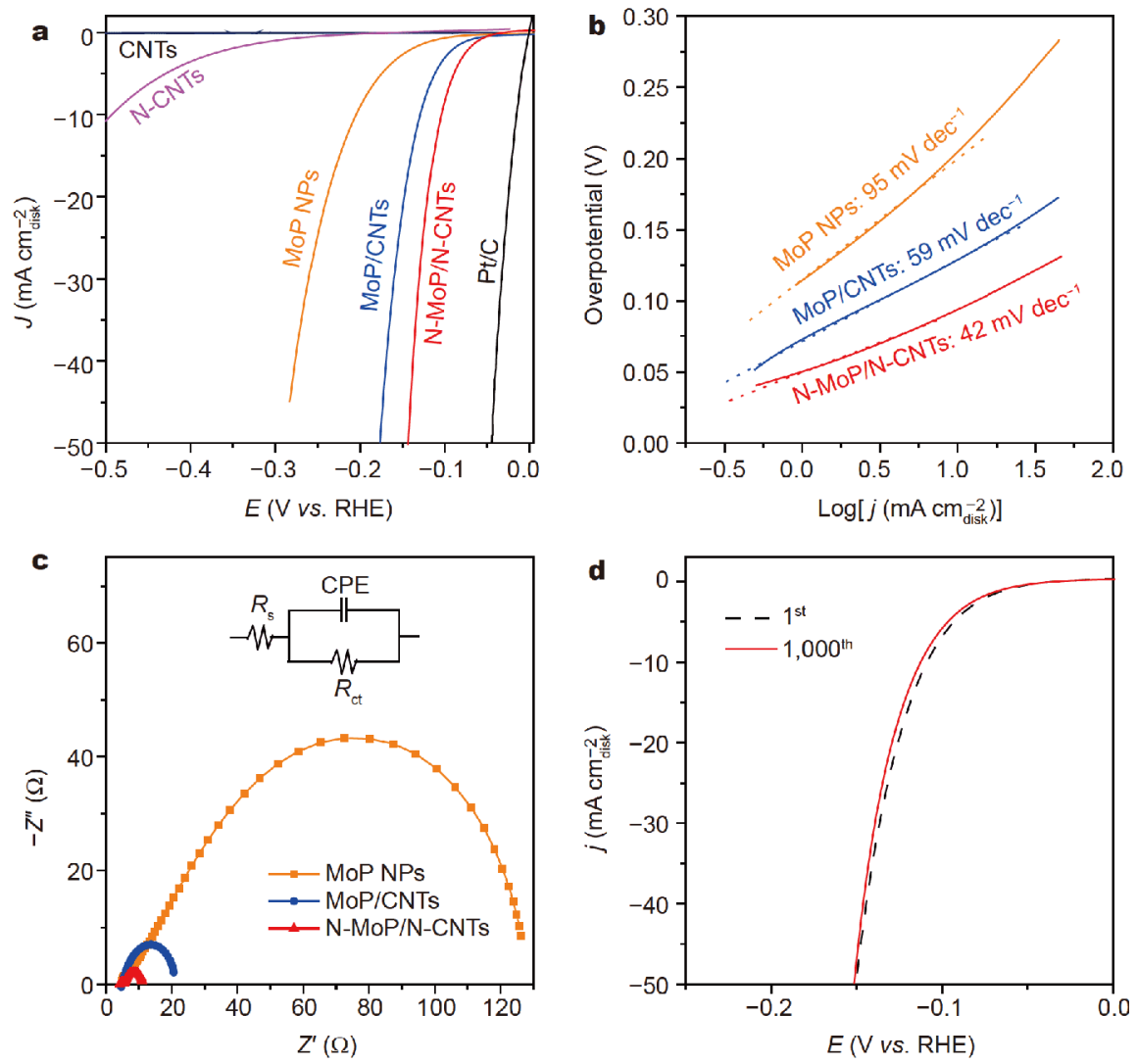

Figure 5 (a) Polarization curves for CNTs, N-CNTs, MoP NPs, MoP/CNTs, N-MoP/N-CNTs and Pt/C in $0.5 \mathrm{~mol} \mathrm{~L}^{-1} \mathrm{H}_{2} \mathrm{SO}_{4}$ at a scan rate of $5 \mathrm{mV} \mathrm{s}^{-1}$. (b) Tafel plots for MoP NPs, MoP/CNTs, N-MoP/N-CNTs. (c) Nyquist plots for MoP NPs, MoP/CNTs, and N-MoP/N-CNTs at an overpotential of $150 \mathrm{mV}$. The inset is the equivalent electrical circuit. (d) LSV polarization curves of N-MoP/N-CNTs initially and after $1,000 \mathrm{CV}$ cycles at a scan rate of $100 \mathrm{mV} \mathrm{s}^{-1}$ between 0 and $-176 \mathrm{mV}$, respectively.

Table 1 Comparison of catalytic performance of different HER electrocatalysts in $0.5 \mathrm{~mol} \mathrm{~L}^{-1} \mathrm{H}_{2} \mathrm{SO}_{4}$

\begin{tabular}{ccc}
\hline Electrocatalyst & $\begin{array}{c}@ 10 \mathrm{~mA} \mathrm{~cm}^{-2} \\
(\mathrm{mV})\end{array}$ & $\begin{array}{c}\text { Tafel slope } \\
\left(\mathrm{mV} \mathrm{dec}^{-1}\right)\end{array}$ \\
\hline $20 \%$ Pt/C & 21 & 30 \\
N-MoP/N-CNTs & 103 & 42 \\
MoP/CNTs & 129 & 59 \\
MoP NPs & 243 & 95 \\
N-CNTs & 493 & 300 \\
\hline
\end{tabular}

parallel doped N-MoP/N-CNTs with the highest HER performance (Fig. S6). Lower temperature is not enough for the phosphidation of $\mathrm{MoO}_{x}$, while higher temperature leads to the aggradation of the MoP NPs.

The hydrogen binding energy is essential for the HER performance. Doped $\mathrm{N}$ atoms modulate the electronic structure of MoP. Doping CNTs with N, the synergistic effect between the NPs and the substrate also adjusts the electronic structure of MoP. The XPS results confirm the positive shift of the Mo $3 \mathrm{~d}$ peaks.

Fig. 5b shows the Tafel plots of the catalysts. The Tafel slope, an inherent property of catalyst, was fitted to the Tafel equation (Equation (4))

$$
\eta=b \log j+a,
$$

where $j$ is the current density and $b$ is the Tafel slope. The

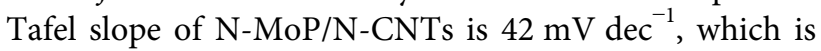
lower than that of MoP/CNTs $\left(59 \mathrm{mV} \mathrm{dec}^{-1}\right.$ ) and MoP NPs (95 mV dec ${ }^{-1}$ ), suggesting that N-MoP/N-CNTs has an effective facilitation on the HER process.

EIS was also conducted to characterize the electrochemical activity. Fig. $5 \mathrm{c}$ shows the EIS spectra of $\mathrm{N}$ MoP/N-CNTs, MoP/CNTs and MoP NPs conducted at an overpotential of $150 \mathrm{mV}$, and the equivalent electrical circuit is shown in the inset of Fig. $5 \mathrm{c}$, where $R_{\mathrm{s}}, R_{\mathrm{ct}}$ and CPE represent the solution resistance, charge transfer resistance and constant phase element, respectively. The $R_{\mathrm{s}}$ of N-MoP/N-CNTs, MoP/CNTs and MoP NPs are $5 \Omega$ 
owing to the same electrolyte. The $R_{\mathrm{ct}}$ for $\mathrm{N}-\mathrm{MoP} /$ $\mathrm{N}$-CNTs is $7 \Omega$, which is lower than that of MoP/CNTs $(16 \Omega)$ and MoP NPs $(120 \Omega)$, suggesting that N-MoP/ $\mathrm{N}-\mathrm{CNTs}$ have faster charge transfer in hydrogen evolution process. For the stability of the as-obtained $\mathrm{N}-\mathrm{MoP} / \mathrm{N}-\mathrm{CNTs}$, we conducted 1,000 continuous cyclic voltammograms (CV) between 0 and $-176 \mathrm{mV}$ with a scan rate of $0.1 \mathrm{~V} \mathrm{~s}^{-1}$. As shown in Fig. $5 \mathrm{~d}$, little activity loss after 1,000 CV cycles indicates the high stability of $\mathrm{N}-\mathrm{MoP} / \mathrm{N}-\mathrm{CNT}$ in acid environment.

All the above results suggest the parallel doping of nitrogen leads to the high HER activity and stability of $\mathrm{N}-\mathrm{MoP} / \mathrm{N}-\mathrm{CNTs}$. In our method, benefiting from the bifunctional phosphidation agent $\mathrm{NH}_{4} \mathrm{H}_{2} \mathrm{PO}_{2}$, the MoP NPs and CNTs are doped with $\mathrm{N}$ at the same time in the phosphidation. This is a general strategy which can be used to synthesize other doped phosphides.

\section{CONCLUSIONS}

In summary, $\mathrm{NH}_{4} \mathrm{H}_{2} \mathrm{PO}_{2}$ was developed as a bifunctional agent for both phosphidation and nitrogen doping, with which parallel doped $\mathrm{N}-\mathrm{MoP} / \mathrm{N}-\mathrm{CNTs}$ were synthesized through a simple process. N doped MoP NPs and CNTs exhibited excellent HER performance in the acid electrolyte. This work developed a facile method to synthesize doped phosphides with promoted HER performance, which sheds light on the design and synthesis of doped catalysts.

\section{Received 19 July 2018; accepted 26 September 2018;} published online 7 November 2018

1 Chow J, Kopp RJ, Portney PR. Energy resources and global development. Science, 2003, 302: 1528-1531

2 Bockris JO. A hydrogen economy. Science, 1972, 176: 1323

3 Bockris JOM. The hydrogen economy: Its history. Int J Hydrogen Energy, 2013, 38: 2579-2588

4 Armaroli N, Balzani V. The future of energy supply: Challenges and opportunities. Angew Chem Int Ed, 2007, 46: 52-66

5 Kudo A, Miseki Y. Heterogeneous photocatalyst materials for water splitting. Chem Soc Rev, 2009, 38: 253-278

6 Luo J, Im JH, Mayer MT, et al. Water photolysis at 12.3\% efficiency via perovskite photovoltaics and Earth-abundant catalysts. Science, 2014, 345: 1593-1596

7 Nocera DG. The artificial leaf. Acc Chem Res, 2012, 45: 767-776

8 McCrory CCL, Jung S, Ferrer IM, et al. Benchmarking hydrogen evolving reaction and oxygen evolving reaction electrocatalysts for solar water splitting devices. J Am Chem Soc, 2015, 137: 4347-4357

9 Nørskov JK, Bligaard T, Logadottir A, et al. Trends in the exchange current for hydrogen evolution. J Electrochem Soc, 2005, 152: J23

10 Yan Y, Xia BY, Xu Z, et al. Recent development of molybdenum sulfides as advanced electrocatalysts for hydrogen evolution reaction. ACS Catal, 2014, 4: 1693-1705

11 Zeng M, Li Y. Recent advances in heterogeneous electrocatalysts for the hydrogen evolution reaction. J Mater Chem A, 2015, 3: 14942-14962

12 Callejas JF, Read CG, Roske CW, et al. Synthesis, characterization, and properties of metal phosphide catalysts for the hydrogenevolution reaction. Chem Mater, 2016, 28: 6017-6044

13 Lu S, Zhuang Z. Electrocatalysts for hydrogen oxidation and evolution reactions. Sci China Mater, 2016, 59: 217-238

14 Shi Y, Zhang B. Recent advances in transition metal phosphide nanomaterials: synthesis and applications in hydrogen evolution reaction. Chem Soc Rev, 2016, 45: 1529-1541

15 Wang J, $\mathrm{Xu} \mathrm{F}$, Jin $\mathrm{H}$, et al. Non-noble metal-based carbon composites in hydrogen evolution reaction: fundamentals to applications. Adv Mater, 2017, 29: 1605838

16 Wang J, Cui W, Liu Q, et al. Recent progress in cobalt-based heterogeneous catalysts for electrochemical water splitting. Adv Mater, 2016, 28: 215-230

17 Popczun EJ, McKone JR, Read CG, et al. Nanostructured nickel phosphide as an electrocatalyst for the hydrogen evolution reaction. J Am Chem Soc, 2013, 135: 9267-9270

18 Jiang $\mathrm{P}$, Liu $\mathrm{Q}$, Liang $\mathrm{Y}$, et al. A cost-effective 3D hydrogen evolution cathode with high catalytic activity: FeP nanowire array as the active phase. Angew Chem Int Ed, 2014, 53: 12855-12859

19 Tian J, Liu Q, Asiri AM, et al. Self-supported nanoporous cobalt phosphide nanowire arrays: an efficient $3 \mathrm{D}$ hydrogen-evolving cathode over the wide range of $\mathrm{pH}$ 0-14. J Am Chem Soc, 2014, 136: 7587-7590

20 Tian J, Liu Q, Cheng N, et al. Self-supported $\mathrm{Cu}_{3} \mathrm{P}$ nanowire arrays as an integrated high-performance three-dimensional cathode for generating hydrogen from water. Angew Chem Int Ed, 2014, 53: 9577-9581

21 Popczun EJ, Read CG, Roske CW, et al. Highly active electrocatalysis of the hydrogen evolution reaction by cobalt phosphide nanoparticles. Angew Chem Int Ed, 2014, 53: 54275430

22 Yang J, Zhang F, Wang X, et al. Porous molybdenum phosphide nano-octahedrons derived from confined phosphorization in UIO66 for efficient hydrogen evolution. Angew Chem Int Ed, 2016, 55: 12854-12858

23 Chen X, Wang D, Wang Z, et al. Molybdenum phosphide: a new highly efficient catalyst for the electrochemical hydrogen evolution reaction. Chem Commun, 2014, 50: 11683-11685

24 McEnaney JM, Crompton JC, Callejas JF, et al. Amorphous molybdenum phosphide nanoparticles for electrocatalytic hydrogen evolution. Chem Mater, 2014, 26: 4826-4831

25 Xiao P, Sk MA, Thia L, et al. Molybdenum phosphide as an efficient electrocatalyst for the hydrogen evolution reaction. Energy Environ Sci, 2014, 7: 2624-2629

26 Xing Z, Liu Q, Asiri AM, et al. Closely interconnected network of molybdenum phosphide nanoparticles: a highly efficient electrocatalyst for generating hydrogen from water. Adv Mater, 2014, 26: 5702-5707

27 McFarland EW, Metiu H. Catalysis by doped oxides. Chem Rev, 2013, 113: 4391-4427

28 Paraknowitsch JP, Thomas A. Doping carbons beyond nitrogen: an overview of advanced heteroatom doped carbons with boron, sulphur and phosphorus for energy applications. Energy Environ Sci, 2013, 6: 2839-2855

29 Pham VP, Yeom GY. Recent advances in doping of molybdenum disulfide: industrial applications and future prospects. Adv Mater, 2016, 28: 9024-9059 
30 Tedstone AA, Lewis DJ, O'Brien P. Synthesis, properties, and applications of transition metal-doped layered transition metal dichalcogenides. Chem Mater, 2016, 28: 1965-1974

$31 \mathrm{Wu}$ G, Santandreu A, Kellogg W, et al. Carbon nanocomposite catalysts for oxygen reduction and evolution reactions: From nitrogen doping to transition-metal addition. Nano Energy, 2016, 29: $83-110$

32 Sun $\mathrm{A}$, Shen $\mathrm{Y}, \mathrm{Wu} \mathrm{Z}$, et al. N-doped MoP nanoparticles for improved hydrogen evolution. Int J Hydrogen Energy, 2017, 42: 14566-14571

33 Kibsgaard J, Jaramillo TF. Molybdenum phosphosulfide: an active, acid-stable, earth-abundant catalyst for the hydrogen evolution reaction. Angew Chem Int Ed, 2014, 53: 14433-14437

34 Anjum MAR, Lee JS. Sulfur and nitrogen dual-doped molybdenum phosphide nanocrystallites as an active and stable hydrogen evolution reaction electrocatalyst in acidic and alkaline media. ACS Catal, 2017, 7: 3030-3038

35 Fields M, Tsai C, Chen LD, et al. Scaling relations for adsorption energies on doped molybdenum phosphide surfaces. ACS Catal, 2017, 7: 2528-2534

36 Liang Y, Wang H, Diao P, et al. Oxygen reduction electrocatalyst based on strongly coupled cobalt oxide nanocrystals and carbon nanotubes. J Am Chem Soc, 2012, 134: 15849-15857

37 Zhuang Z, Giles SA, Zheng J, et al. Nickel supported on nitrogendoped carbon nanotubes as hydrogen oxidation reaction catalyst in alkaline electrolyte. Nat Commun, 2016, 7: 10141

38 Wang H, Dai H. Strongly coupled inorganic-nano-carbon hybrid materials for energy storage. Chem Soc Rev, 2013, 42: 3088-3113

$39 \mathrm{Pu}$ Z, Amiinu IS, Liu X, et al. Ultrastable nitrogen-doped carbon encapsulating molybdenum phosphide nanoparticles as highly efficient electrocatalyst for hydrogen generation. Nanoscale, 2016, 8: 17256-17261

40 Zhao Y, Wang S, Li C, et al. Nanostructured molybdenum phosphide/N, P dual-doped carbon nanotube composite as electrocatalysts for hydrogen evolution. RSC Adv, 2016, 6: 73707377

41 Song J, Xiang J, Mu C, et al. Facile synthesis and excellent electrochemical performance of CoP nanowire on carbon cloth as bifunctional electrode for hydrogen evolution reaction and supercapacitor. Sci China Mater, 2017, 60: 1179-1186

42 Ellison MD, Crotty MJ, Koh D, et al. Adsorption of $\mathrm{NH}_{3}$ and $\mathrm{NO}_{2}$ on single-walled carbon nanotubes. J Phys Chem B, 2004, 108: 7938-7943

43 Yi H, Yu Q, Tang X, et al. Phosphine adsorption removal from yellow phosphorus tail gas over $\mathrm{CuO}-\mathrm{ZnO}-\mathrm{La}_{2} \mathrm{O}_{3} /$ activated carbon. Ind Eng Chem Res, 2011, 50: 3960-3965

44 Phillips DC, Sawhill SJ, Self R, et al. Synthesis, characterization, and hydrodesulfurization properties of silica-supported molybdenum phosphide catalysts. J Catal, 2002, 207: 266-273

45 Bai J, Li X, Wang A, et al. Different role of $\mathrm{H}_{2} \mathrm{~S}$ and dibenzothiophene in the incorporation of sulfur in the surface of bulk MoP during hydrodesulfurization. J Catal, 2013, 300: 197-200

46 Sanjinés R, Wiemer C, Almeida $\mathrm{J}$, et al. Valence band photoemission study of the $\mathrm{Ti}_{1-x} \mathrm{Mo}_{x} \mathrm{~N}_{y}$ system. Thin Solid Films, 1996, 290-291: 334-338

47 Zhang J, Jiang J, Zhao XS. Synthesis and capacitive properties of manganese oxide nanosheets dispersed on functionalized graphene sheets. J Phys Chem C, 2011, 115: 6448-6454

Acknowledgements This work was supported by the National Key Research and Development Program of China (2017YFA0206500), the National Natural Science Foundation of China (21671014) and the Fundamental Research Funds for the Central Universities (buctrc201522).

Author contributions Zhuang Z and Zhang J conceived and designed the experiments. Zhang J performed the experiments with help from Sui $\mathrm{R}$, Xue Y, Wang X and Pei J. Zhang J, Zhuang Z and Liang X wrote the paper. All authors contributed to the general discussion.

Conflict of interest The authors declare no conflict of interest.

Supplementary information Supporting data are available in the online version of the paper. 


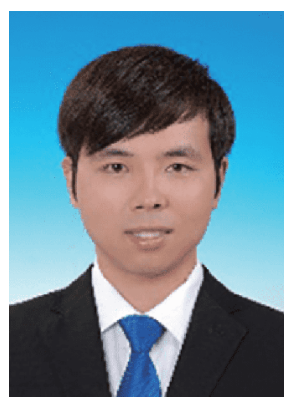

Juntao Zhang received his BSc degree from Liaoning Shihua University in 2013 and his MSc degree from Beijing University of Chemical Technology in 2016. He is now a PhD candidate in Beijing University of Chemical Technology under the supervision of Prof. Zhongbin Zhuang. His research interest focuses on the materials for electrocatalysis.

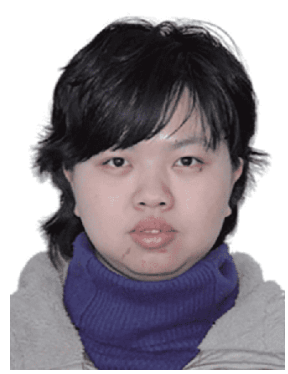

Xin Liang is an associate professor at Beijing University of Chemical Technology. She received her PhD degree from Tsinghua University in 2009. She worked in the University of California, Santa Barbara from 2013 to 2014 as a research fellow. Her research interest includes design, synthesis and characterization of functional catalytic materials towards industrial and energy catalysis, such as hydrodesulfurization, hydrogenations and electrocatalysis.

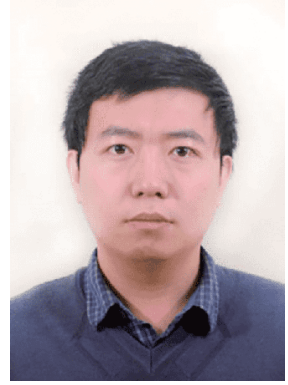

Zhongbin Zhuang received his $\mathrm{PhD}$ degrees from Tsinghua University in 2010. After postdoctoral work at the University of California, Riverside and University of Delaware, he joined Beijing University of Chemical Technology as a professor in 2015. His current research interest includes electrocatalysts for fuel cells and electrolyzers, interfacial electrochemistry and methodology for nanocrystal synthesis.

\section{直接合成双掺杂N-MoP/N-CNT及其高效析氢催化性能}

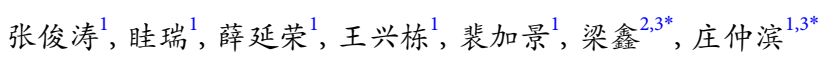

摘要 清洁氢能源是未来发展的重要方向, 因此开发高效廉价的析氢材料尤为重要. 掺杂的磷化物作为一种优异的析氢材料得到了广泛 的关注. 本文提出了一种将氮原子同时掺杂在磷化物催化剂和载体上的新的合成方法. 在热处理的过程中, 利用次磷酸铵 $\left(\mathrm{NH}_{4} \mathrm{H}_{2} \mathrm{PO}_{2}\right)$ 分 解产生的氨和磷化氢气体与前驱体进行反应, 一步得到双掺杂的氮掺杂碳纳米管负载氮掺杂磷化钼催化剂 $(\mathrm{N}-\mathrm{MoP} / \mathrm{N}-\mathrm{CNT})$. 该催化剂展 现出了良好的析氢反应活性, 当电流密度为 $10 \mathrm{~mA} \mathrm{~cm}^{-2}$ 时, 过电势只有 $103 \pm 5 \mathrm{mV}$, 明显低于 $\mathrm{MoP}$ 纳米颗粒的过电势( $243 \mathrm{mV}$. 催化活性的 提升主要来自掺氮带来的电子效应以及协同效应. 该催化剂在电解水产氢方面具有应用前景. 